Supplementary Material

\title{
Aryl Annulation of Cyclic Ketones via a Magnesium
}

\section{Carbometallation-6- $\pi$-Electrocyclization Protocol}

Pierre E. Tessier, ${ }^{\dagger}$ Natalie Nguyen, Matthew Clay, and Alex G. Fallis*

Department of Chemistry, University of Ottawa, 10 Marie Curie,

Ottawa, Ontario, Canada K1N 6N5

afallis@science.uottawa.ca

2-Trifluoromethanesulfonylbicyclo[2.2.1]heptene (7) ${ }^{3}$ : $n$-Butyllithium $(6.4 \mathrm{~mL}, 2.5 \mathrm{M}, 16.0$ $\mathrm{mmol})$, diisopropylamine $(2.10 \mathrm{~mL}, 16.0 \mathrm{mmol})$, norcamphor $(1.6 \mathrm{~g}, 14.5 \mathrm{mmol})$ in THF $(5 \mathrm{~mL})$, $N$-phenyltrifluromethanesulfonimide $(5.45 \mathrm{~g}, 15.3 \mathrm{mmol})$ were reacted in THF $(10 \mathrm{~mL})$. Chromatography (100\% petroleum ether) afforded 7 as a colourless oil $(2.06 \mathrm{~g}, 59 \%)$. ${ }^{1} \mathrm{H}$ NMR $\left(\mathrm{CDCl}_{3}, 300 \mathrm{MHz}\right): \delta 5.65(\mathrm{~d}, J=3.5 \mathrm{~Hz}, 1 \mathrm{H}), 2.98-2.96(\mathrm{~m}, 2 \mathrm{H}), 1.82-1.69(\mathrm{~m}, 2 \mathrm{H}), 1.67-$ $1.61(\mathrm{~m}, 1 \mathrm{H}), 1.49-1.34(\mathrm{~m}, 1 \mathrm{H}), 1.25-1.13(\mathrm{~m}, 3 \mathrm{H}), 0.89-0.79(\mathrm{~m}, 2 \mathrm{H}) ;{ }^{13} \mathrm{C} \mathrm{NMR}\left(\mathrm{CDCl}_{3}\right.$, $75 \mathrm{MHz}): \delta 155.3,125.3,121.1,120.3,116.8,112.6,48.0,44.1,41.7,25.9,24.6$.

Trifluoromethanesulfony-1,7,7-trimethylbicyclo[2.2.1]hept-2-ene $(\mathbf{1 0})^{\mathbf{4}}$ : Diisopropylamine $(8.10 \mathrm{~mL}, 57.8 \mathrm{mmol})$ was dissolved in THF $(125 \mathrm{~mL})$, cooled to $-78{ }^{\circ} \mathrm{C}$ and a solution of $n$ BuLi in hexane $(2.5 \mathrm{M}, 23.1 \mathrm{~mL}, 57.8 \mathrm{mmol})$ was added. The solution was stirred for 15 minutes, and then a solution of camphor $(8.00 \mathrm{~g}, 52.6 \mathrm{mmol})$ in THF $(75 \mathrm{~mL})$ was added. After stirring at $-78^{\circ} \mathrm{C}$ for 90 minutes, a solution of $\operatorname{PhNTf}_{2}(19.7 \mathrm{~g}, 55.2 \mathrm{mmol})$ in THF $(75 \mathrm{~mL})$ was added and the reaction was stirred at $-78{ }^{\circ} \mathrm{C}$ for 15 minutes and then at $0{ }^{\circ} \mathrm{C}$ for 1 hour. The reaction was concentrated to $\sim 100 \mathrm{~mL}$ on the rotary evaporator $\left(\mathrm{T}=40{ }^{\circ} \mathrm{C}\right)$, diluted with water $(200 \mathrm{~mL})$, and extracted with ether $(3 \times 200 \mathrm{~mL})$. The combined organics were dried $\left(\mathrm{MgSO}_{4}\right)$, filtered, and carefully concentrated (CAUTION: Product is volatile!). Column chromatography (petroleum ether) afforded the title compound as a clear colorless oil $(13.30 \mathrm{~g}, 89 \%)$. ${ }^{1} \mathrm{H}$ NMR $\left(500 \mathrm{MHz}, \mathrm{CDCl}_{3}\right) \delta 5.67(\mathrm{~d}, J=3.8 \mathrm{~Hz}, 1 \mathrm{H}), 2.46(\mathrm{dd}, J=3.8$ and $3.8 \mathrm{~Hz}, 1 \mathrm{H}), 1.91-1.97(\mathrm{~m}$, $1 \mathrm{H}), 1.66(\mathrm{ddd}, J=12.2,8.6$ and $3.7 \mathrm{~Hz}, 1 \mathrm{H}), 1.34(\mathrm{ddd}, J=12.4,9.1$ and $3.7 \mathrm{~Hz}, 1 \mathrm{H}), 1.16$ (ddd, $J=12.5,9.1$ and $3.7 \mathrm{~Hz}, 1 \mathrm{H}), 1.04(\mathrm{~s}, 3 \mathrm{H}), 0.93(\mathrm{~s}, 3 \mathrm{H}), 0.80(\mathrm{~s}, 3 \mathrm{H}) ;{ }^{13} \mathrm{C} \mathrm{NMR}(125 \mathrm{MHz}$, $\left.\mathrm{CDCl}_{3}\right) \delta 155.2,118.6,117.7,56.9,53.8,50.1,30.8,25.3,19.6,18.9,9.3$; IR (neat): $v=2964$, 2880, 1624, 1424, 1321, 1212, 1142, 1111, 1085, 1004, 914, 880, 813, $755 \mathrm{~cm}^{-1}$; MS (EI) $\mathrm{m} / \mathrm{z}$ $284\left(\mathrm{M}^{+}\right)$(42), 269 (14), 151 (38), 123 (100), 95 (62), 55 (59), 41 (77); HRMS calculated for $\mathrm{C}_{11} \mathrm{H}_{15} \mathrm{O}_{3} \mathrm{~F}_{3} \mathrm{~S}\left(\mathrm{M}^{+}\right)$284.0694, found 284.0703.

Trifluoromethanesulfonyl-7,7-dimethylbicyclo[3.1.1]hept-2-ene (13): $n$-Butyllithium (9.3 $\mathrm{mL}, 1.6 \mathrm{M}, 14.8 \mathrm{mmol})$, diisopropylamine $(1.94 \mathrm{~mL}, 14.8 \mathrm{mmol})$, nopinone $(1.9 \mathrm{~mL}, 13.46$ mmol), $N$-phenyltrifluromethanesulfonimide $(5.05 \mathrm{~g}, 14.13 \mathrm{mmol})$ were reacted in THF $(10 \mathrm{~mL})$. Chromatography (100\% ether) afforded 13 as a colourless oil (1.94 g, 54\%). IR (neat); 2949 (s), 
$1658(\mathrm{~m}), 1414(\mathrm{~s}), 1224(\mathrm{~s}) \mathrm{cm}^{-1} ;{ }^{1} \mathrm{H}$ NMR $\left(\mathrm{CDCl}_{3}, 300 \mathrm{MHz}\right): \delta 5.51(\mathrm{q}, J=3.3 \mathrm{~Hz}, 1 \mathrm{H}), 2.54$ $(\mathrm{dt}, J=9.2 \mathrm{~Hz}, 5.6 \mathrm{~Hz}, 1 \mathrm{H}), 2.22-2.40(\mathrm{~m}, 3 \mathrm{H}), 2.09-2.13(\mathrm{~m}, 1 \mathrm{H}), 1.35(\mathrm{~d}, J=9.1 \mathrm{~Hz}, 1 \mathrm{H})$, $1.32(\mathrm{~s}, 3 \mathrm{H}), 0.91(\mathrm{~s}, 3 \mathrm{H}) ;{ }^{13} \mathrm{C} \mathrm{NMR}\left(\mathrm{CDCl}_{3}, 75 \mathrm{MHz}\right): \delta 155.4,125.2,121.0,116.8,112.6$, 111.8, 46.6, 40.5, 40.1, 32.1, 28.6, 25.8, 21.2; MS (EI) m/z $270.1\left(\mathrm{M}^{+}\right.$(2.6), 226.0 (16.2), 162.0 (9.0), 77.0 (52.2), 55.0 (100.0); Exact mass calc'd. for $\mathrm{C}_{10} \mathrm{H}_{13} \mathrm{~F}_{3} \mathrm{O}_{3} \mathrm{~S}: 270.0517$, found 270.0496.

2-Trifluoromethanesulfonylbicyclo[3.2.1] oct-2-ene (16): $n$-Butyllithium (7.0 mL, $2.5 \mathrm{M}, 17.6$ $\mathrm{mmol})$, diisopropylamine $(2.30 \mathrm{~mL}, 17.6 \mathrm{mmol})$, bicyclo [3.2.1] octanone (1.99 $\mathrm{g}, 16.0 \mathrm{mmol})$ in THF (5 mL), $N$-phenyltrifluromethanesulfonimide $(6.0 \mathrm{~g}, 16.8 \mathrm{mmol})$ were reacted in THF (10 $\mathrm{mL})$. Chromatography (100\% petroleum ether) afforded 16 as a colourless $(3.55 \mathrm{~g}, 87 \%)$. IR (neat); 2955 (s), 2865 (s), $1416(\mathrm{~s}), 1210$ (s) cm ${ }^{-1} ;{ }^{1} \mathrm{H}$ NMR $\left(\mathrm{CDCl}_{3}, 300 \mathrm{MHz}\right): \delta 5.40$ - 5.38 (m, $1 \mathrm{H}), 2.53(\mathrm{t}, J=4.7 \mathrm{~Hz}, 1 \mathrm{H}), 2.42-2.36(\mathrm{~m}, 2 \mathrm{H}), 2.15-2.26(\mathrm{~m}, 1 \mathrm{H}), 1.96-1.84(\mathrm{~m}, 2 \mathrm{H}), 1.68$ $-1.80(\mathrm{~m}, 2 \mathrm{H}), 1.62-1.54(\mathrm{~m}, 1 \mathrm{H}), 1.47-1.38(\mathrm{~m}, 1 \mathrm{H}) ;{ }^{13} \mathrm{C} \mathrm{NMR}\left(\mathrm{CDCl}_{3}, 75 \mathrm{MHz}\right): \delta 154.2$, 125.2, 121.0, 116.8, 112.6, 114.1, 40.0, 36.0, 34.8, 34.4, 32.5, 30.3; MS (EI) m/z $256.0\left(\mathrm{M}^{+}\right.$ (44.2), 228.0 (20.3), 95.1 (100.0), 79.1 (57.4), 67.1 (74.8)); Exact mass calc'd. for $\mathrm{C}_{9} \mathrm{H}_{11} \mathrm{~F}_{3} \mathrm{O}_{3} \mathrm{~S}$ : 256.0379 , found 256.0376 .

1-Trifluoromethanesulfony-5-methoxy-3,4-dihydronaphthalene (34) $n$ - Butyllithium (6.0 $\mathrm{mL}, 2.5 \mathrm{M}, 14.6 \mathrm{mmol})$, diisopropylamine $(1.92 \mathrm{~mL}, 14.6 \mathrm{mmol}), 6$-methoxy tetralone $(2.35 \mathrm{~g}$, $13.3 \mathrm{mmol})$ in THF $(5 \mathrm{~mL}), N$-phenyltrifluromethanesulfonimide $(5.05 \mathrm{~g}, 14.13 \mathrm{mmol})$ were reacted in THF $(10 \mathrm{~mL})$. Chromatography (petroleum ether/ethyl acetate, 19:1) afforded 36 as beige oil (3.30 g, 82\%). IR (neat); 2930 (s), 2724 (m), 1693 (s), 1608 (m), 1382 (m), 1230 (m) $\mathrm{cm}^{-1} ;{ }^{1} \mathrm{H} \mathrm{NMR}\left(\mathrm{CDCl}_{3}, 300 \mathrm{MHz}\right): \delta 7.27(\mathrm{~d}, J=8.4 \mathrm{~Hz}, 1 \mathrm{H}), 6.78-6.69(\mathrm{~m}, 2 \mathrm{H}), 5.85(\mathrm{t}, J=$ $4.8 \mathrm{~Hz}, 1 \mathrm{H}), 3.79(\mathrm{~s}, 3 \mathrm{H}), 2.82(\mathrm{t}, J=8.0 \mathrm{~Hz}, 2 \mathrm{H}), 2.47(\mathrm{dt}, J=4.6 \mathrm{~Hz}, 8.3 \mathrm{~Hz}, 2 \mathrm{H}) ;{ }^{13} \mathrm{C} \mathrm{NMR}$ $\left(\mathrm{CDCl}_{3}, 75 \mathrm{MHz}\right): \delta 160.6,146.7,138.7,131.3,125.5,123.1,121.9,121.1,116.9,115.1,114.6$, 112.6, 111.7, 55.6, 27.7, 22.6; MS (EI) m/z $308.0\left(\mathrm{M}^{+}\right.$(70.6), 175.1 (41.1), 147.1 (100.0), 115.1 (37.9), 77.0 (28.8)); Exact mass calc'd. for $\mathrm{C}_{12} \mathrm{H}_{11} \mathrm{~F}_{3} \mathrm{O}_{4} \mathrm{~S}$ : 308.0328, found 308.0326.

1-Trifluoromethanesulfony-3,4-dihydro-naphthalene $(37)^{5}: n$-Butyllithium $(7.0 \mathrm{~mL}, 2.4 \mathrm{M}$, $16.7 \mathrm{mmol})$, diisopropylamine $(2.18 \mathrm{~mL}, 16.7 \mathrm{mmol})$, tetralone $(2.02 \mathrm{~mL}, 15.2 \mathrm{mmol})$ in THF $(5$ $\mathrm{mL}), \mathrm{N}$-phenyltrifluromethanesulfonimide $(5.7 \mathrm{~g}, 16.0 \mathrm{mmol})$ were reacted in THF $(10 \mathrm{~mL})$. Chromatography (petroleum ether) afforded $37 \mathrm{~s}$ a colourless oil $(6.11 \mathrm{~g}, 82 \%)$. ${ }^{1} \mathrm{H}$ NMR $\left(\mathrm{CDCl}_{3}, 500 \mathrm{MHz}\right): \delta 7.37-7.35(\mathrm{~m}, 1 \mathrm{H}), 7.27-7.25(\mathrm{~m}, 2 \mathrm{H}), 7.18-7.17(\mathrm{~m}, 1 \mathrm{H}), 6.02(\mathrm{t}, J=$ $4.8 \mathrm{~Hz}, 1 \mathrm{H}), 2.86(\mathrm{t}, J=8.0 \mathrm{~Hz}, 2 \mathrm{H}), 2.52-2.48(\mathrm{~m}, 2 \mathrm{H}) ;{ }^{13} \mathrm{C} \mathrm{NMR}\left(\mathrm{CDCl}_{3}, 125 \mathrm{MHz}\right)$ : $\delta 146.4,136.2,129.1,128.6,127.7,126.9,122.4,121.2,119.9,117.7,117.4,26.8,22.3$.

1-Trifluoromethanesulfonycyclohex-1-ene (40) ${ }^{6}: n$-Butyllithium $(5.96 \mathrm{~mL}, 2.5 \mathrm{M}, 14.9 \mathrm{mmol})$, diisopropylamine $(1.95 \mathrm{~mL}, 14.9 \mathrm{mmol})$, cyclohexanone $(1.4 \mathrm{~mL}, 13.5 \mathrm{mmol}), N-$ phenyltrifluromethanesulfonimide $(5.1 \mathrm{~g}, 14.2 \mathrm{mmol})$ in THF $(10 \mathrm{~mL})$. Chromatrography $(100 \%$ petroleum ether) afforded 40 as a colourless oil (1.72 g, $55 \%) .{ }^{1} \mathrm{H}$ NMR $\left(\mathrm{CDCl}_{3}, 200 \mathrm{MHz}\right)$ : $\delta 5.73(\mathrm{t}, J=3.8 \mathrm{~Hz}, 1 \mathrm{H}), 2.29-2.20(\mathrm{~m}, 2 \mathrm{H}), 2.17-2.13(\mathrm{~m}, 2 \mathrm{H}), 1.82-1.70(\mathrm{~m}, 2 \mathrm{H}), 1.63-$ $1.54(\mathrm{~m}, 2 \mathrm{H}) ;{ }^{13} \mathrm{C} \mathrm{NMR}\left(\mathrm{CDCl}_{3}, 50 \mathrm{MHz}\right): \delta 149.3,128.0,121.7,118.3,115.3,108.9,27.3,23.6$, 22.4, 20.7.

Sonogashira Coupling of Propargyl Alcohol to a Vinyl Triflate: $\mathrm{Pd}\left(\mathrm{PPh}_{3}\right)_{2} \mathrm{Cl}_{2}(0.05$ eq) and copper (I) iodide $(0.1$ eq) were dissolved in a 1:1 solution of THF:DEA (diethylamine). The 
solution was degassed with $\mathrm{N}_{2}$ for 10 minutes. The appropriate vinyl triflate (1 eq) was dissolved in THF and the solution degassed with $\mathrm{N}_{2}$ for an additional 10 minutes. Propargyl alcohol (1.1 eq) was added, via syringe, and the initial green solution changed from yellow, to orange to red. The solution was refluxed for 16 hours. The THF and DEA were removed under reduced pressure (rotary evaporator). Purification was achieved by flash silica gel chromatography with an appropriate petroleum ether/ethyl acetate solvent mixture.

3-Bicyclo[2.2.1]hept-2-yl-prop-2-yn-1-ol (8): $\mathrm{Pd}\left(\mathrm{PPh}_{3}\right)_{2} \mathrm{Cl}_{2}$ (269 mg, $\left.0.38 \mathrm{mmol}\right)$, copper (I) iodide (145 mg, $0.76 \mathrm{mmol})$, THF:DEA $(50 \mathrm{~mL})$, (7) $(1.85 \mathrm{~g}, 7.65 \mathrm{mmol})$ in THF $(10 \mathrm{~mL})$, and propargyl alcohol $(0.49 \mathrm{~mL}, 8.42 \mathrm{mmol})$ were reacted together. Chromatography (petroleum ether/ethyl acetate 5:1) afforded 8 as a viscous red oil $(680 \mathrm{mg}, 60 \%)$. IR $\left(\mathrm{CDCl}_{3}\right) ; 3606(\mathrm{~s})$,

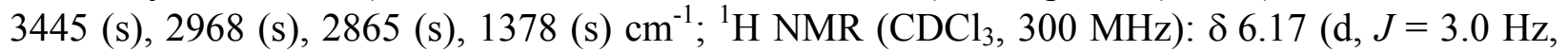
$1 \mathrm{H}), 4.32(\mathrm{~s}, 2 \mathrm{H}), 3.24$ (br s, $1 \mathrm{H}), 2.82-2.80(\mathrm{~m}, 2 \mathrm{H}), 1.67-1.52(\mathrm{~m}, 3 \mathrm{H}), 1.39-1.33(\mathrm{~m}, 2 \mathrm{H})$, $1.19-0.94(\mathrm{~m}, 4 \mathrm{H}) ;{ }^{13} \mathrm{C} \mathrm{NMR}\left(\mathrm{CDCl}_{3}, 75 \mathrm{MHz}\right): \delta 142.1,129.3,90.7,82.4,51.7,48.4,47.3$, 43.0, 25.5, 24.7; MS (EI) m/z 148.1 ( $\mathrm{M}^{+}$(37.6), 120.1 (63.9), 91.1 (100.0), 77.0 (33.8); Exact mass calc'd. for $\mathrm{C}_{12} \mathrm{H}_{16} \mathrm{O}$ : 176.1183 , found 176.1164 .

(1,7,7-Trimethyl-bicyclo[2.2.1]hept-2-en-2-yl)-prop-2-yn-1-ol (11): Triflate 10 (2.00 g, 7.03 $\mathrm{mmol}), \mathrm{CuI}(0.13 \mathrm{~g}, 0.70 \mathrm{mmol}), \mathrm{Pd}\left(\mathrm{PPh}_{3}\right)_{2} \mathrm{Cl}_{2}(0.25 \mathrm{~g}, 0.35 \mathrm{mmol}), \mathrm{Et}_{2} \mathrm{NH}(20 \mathrm{~mL})$ and $\mathrm{THF}$ $(20 \mathrm{~mL})$ were added to a flask and degassed with argon for 20 minutes. Propargyl alcohol $(0.49$ $\mathrm{mL}, 8.44 \mathrm{mmol}$ ) was added and the reaction was heated at reflux overnight. The next day the reaction was cooled to room temperature and silica gel $(\sim 3 \mathrm{~g})$ was added. After removal of the solvent, dry-pack column chromatography (9:1, petroleum ether:ethyl acetate) afforded the title compound as a yellow oil that solidified upon refrigeration $(1.30 \mathrm{~g}, 97 \%)$. $\mathrm{mp}<20{ }^{\circ} \mathrm{C} .{ }^{1} \mathrm{H}$ NMR $\left(500 \mathrm{MHz}, \mathrm{CDCl}_{3}\right) \delta 6.27(\mathrm{~d}, J=3.4 \mathrm{~Hz}, 1 \mathrm{H}), 4.43(\mathrm{~s}, 2 \mathrm{H}), 2.34(\mathrm{dd}, J=3.6$ and $3.6 \mathrm{~Hz}$, $1 \mathrm{H}), 1.88(\mathrm{~m}, 2 \mathrm{H}), 1.55(\mathrm{ddd}, J=11.9,9.1$ and $3.4 \mathrm{~Hz}, 1 \mathrm{H}), 1.07(\mathrm{~m}, 2 \mathrm{H}), 1.05(\mathrm{~s}, 3 \mathrm{H}), 1.00$ (ddd, $J=12.4,9.1$ and $3.4 \mathrm{~Hz}, 1 \mathrm{H}), 0.79_{1}(\mathrm{~s}, 3 \mathrm{H}), 0.78_{7}(\mathrm{~s}, 3 \mathrm{H}) ;{ }^{13} \mathrm{C} \mathrm{NMR}\left(125 \mathrm{MHz}, \mathrm{CDCl}_{3}\right) \delta$ $141.4,131.7,91.1,82.2,60.6,56.7,55.8,52.3,52.0,31.3,25.0,19.8,19.6,12.0$; IR (neat): $v=$ 3329, 3062, 2955, 2871, 2214, 1387, 1366, 1291, 1141, 1026, 1005, $825 \mathrm{~cm}^{-1}$; MS (EI) $\mathrm{m} / z 190$ $\left(\mathrm{M}^{+}\right)$(100), 175 (66), 162 (59), 147 (63), 129 (36), 115 (48), 105 (37), 91 (73), 77 (48), 41 (66); HRMS calculated for $\mathrm{C}_{13} \mathrm{H}_{18} \mathrm{O}\left(\mathrm{M}^{+}\right)$190.1358, found 190.1349 .

(Bicyclo[3.2.1]oct-2-en-2-yl)-prop-2-yn-1-ol (17): $\mathrm{Pd}\left(\mathrm{PPh}_{3}\right)_{2} \mathrm{Cl}_{2}$ (344 mg, $0.49 \mathrm{mmol}$ ), copper (I) iodide $(187 \mathrm{mg}, 0.98 \mathrm{mmol})$, THF:DEA $(80 \mathrm{~mL}), \mathbf{1 6},(2.50 \mathrm{~g}, 9.80 \mathrm{mmol})$ in THF $(5 \mathrm{~mL})$, and propargyl alcohol $(0.63 \mathrm{~mL}, 10.8 \mathrm{mmol})$ were reacted together. Chromatography (neutral alumina gel (petroleum ether/ethyl acetate 7:1) afforded 17 as a viscous orange oil (1.03 g, 65\%). IR (neat); 3341 (s), 2942 (s), $2865(\mathrm{~m}), 1029(\mathrm{~m}) \mathrm{cm}^{-1} ;{ }^{1} \mathrm{H} \mathrm{NMR}\left(\mathrm{CDCl}_{3}, 300 \mathrm{MHz}\right): \delta 5.72(\mathrm{t}, J$ $=3.1 \mathrm{~Hz}, 1 \mathrm{H}), 4.30(\mathrm{~s}, 2 \mathrm{H}), 3.05(\mathrm{br} \mathrm{s}, 1 \mathrm{H}), 2.39-2.23(\mathrm{~m}, 3 \mathrm{H}), 1.89-1.72(\mathrm{~m}, 3 \mathrm{H}), 1.69-$ $1.61(\mathrm{~m}, 1 \mathrm{H}), 1.56-1.52(\mathrm{~m}, 1 \mathrm{H}), 1.45-1.30(\mathrm{~m}, 3 \mathrm{H}) ;{ }^{13} \mathrm{C} \mathrm{NMR}\left(\mathrm{CDCl}_{3}, 75 \mathrm{MHz}\right): \delta 132.2$, $128.3,86.9,85.8,51.6,40.7,37.7,35.5,35.3,32.8$ 30.9; MS (EI) m/z 162.1 (M+ (100.0), 134.1 (26.9), 105.1 (33.8), 91.1 (67.7), 77.0 (45.8)); Exact mass calc'd. for $\mathrm{C}_{11} \mathrm{H}_{14} \mathrm{O}$ : 162.1037 , found 162.1030 .

(5-Methoxy-3,4-dihydro-naphthalen-1-yl)-prop-2-yn-1-ol (35): $\mathrm{Pd}\left(\mathrm{PPh}_{3}\right)_{2} \mathrm{Cl}_{2}(204 \mathrm{mg}, 0.29$ mmol), copper (I) iodide (110 mg, $0.58 \mathrm{mmol})$, THF:DEA $(50 \mathrm{~mL}),(34)(1.76 \mathrm{mg}, 5.8 \mathrm{mmol})$ in THF (3 mL), and propargyl alcohol $(0.37 \mathrm{~mL}, 6.3 \mathrm{mmol})$ were reacted together. Chromatography 
(petroleum ether/ethyl acetate, 7:1) afforded 35 as a viscous red oil (600 mg, 53\%). IR (neat); 3405 (s), 2944 (s), 2842 (s), 1690 (m) cm ${ }^{-1} ;{ }^{1} \mathrm{H}$ NMR $\left(\mathrm{CDCl}_{3}, 300 \mathrm{MHz}\right): \delta 7.45$ (d, J = 8.4 Hz, $1 \mathrm{H}), 6.71(\mathrm{dd}, J=8.4 \mathrm{~Hz}, 2.6 \mathrm{~Hz}, 1 \mathrm{H}), 6.65(\mathrm{~d}, J=2.6 \mathrm{~Hz}, 1 \mathrm{H}), 6.31(\mathrm{t}, J=4.8 \mathrm{~Hz}, 1 \mathrm{H}), 4.48(\mathrm{~s}$, 2H), $3.78(\mathrm{~s}, 3 \mathrm{H}), 2.73(\mathrm{t}, J=8.0 \mathrm{~Hz}, 2 \mathrm{H}), 2.36-2.29(\mathrm{~m}, 2 \mathrm{H}), 2.29(\mathrm{br} s, 1 \mathrm{H}) ;{ }^{13} \mathrm{C} \mathrm{NMR}$ $\left(\mathrm{CDCl}_{3}, 75 \mathrm{MHz}\right): \delta 159.5,137.1,133.9,126.6,126.0,121.0,113.9,111.5,88.3,84.1,55.7$, 52.0, 28.0, 23.9; MS (EI) m/z $214.1\left(\mathrm{M}^{+}\right.$(100.0), 171.1 (12.9), 152.1 (17.4), 115.1 (17.3), 63.0 (7.6)); Exact mass calc'd. for $\mathrm{C}_{14} \mathrm{H}_{14} \mathrm{O}_{2}$ : 214.0982, found 214.0971.

(3,4-Dihydro-naphthalen-1-yl)-prop-2-yn-1-ol (38): $\mathrm{Pd}\left(\mathrm{PPh}_{3}\right)_{2} \mathrm{Cl}_{2} \quad(428 \mathrm{mg}, 0.61 \mathrm{mmol})$, copper (I) iodide (232 mg, $1.22 \mathrm{mmol})$, THF:DEA (50 mL), Compound 37 (3.4 g, $12.2 \mathrm{mmol})$ in THF $(10 \mathrm{~mL})$, and propargyl alcohol $(0.71 \mathrm{~mL}, 12.2 \mathrm{mmol})$ were reacted together. Chromatography (petroleum ether/ethyl acetate, 7:1) afforded $\mathbf{3 8}$ as a viscous red oil (1.19 g, 53\%). IR (neat); 3359 (s), 2935 (s), 2830 (s), 1487 (m) cm ${ }^{-1} ;{ }^{1} \mathrm{H} \mathrm{NMR}\left(\mathrm{CDCl}_{3}, 500 \mathrm{MHz}\right)$ : $\delta 7.59(\mathrm{~d}, J=7.5 \mathrm{~Hz}, 1 \mathrm{H}), 7.23(\mathrm{td}, J=7.4 \mathrm{~Hz}, 0.8 \mathrm{~Hz}, 1 \mathrm{H}), 7.18(\mathrm{td}, J=7.4 \mathrm{~Hz}, 1.4 \mathrm{~Hz}, 1 \mathrm{H})$, $7.10(\mathrm{~d}, J=7.3 \mathrm{~Hz}, 1 \mathrm{H}), 6.47(\mathrm{t}, J=4.8 \mathrm{~Hz}, 1 \mathrm{H}), 4.52(\mathrm{~s}, 2 \mathrm{H}), 2.78(\mathrm{t}, J=7.9 \mathrm{~Hz}, 2 \mathrm{H}), 2.75(\mathrm{br}$ $\mathrm{s}, 1 \mathrm{H}), 2.38-2.34(\mathrm{~m}, 2 \mathrm{H}) ;{ }^{13} \mathrm{C} \mathrm{NMR}\left(\mathrm{CDCl}_{3}, 125 \mathrm{MHz}\right): \delta 135.8,134.8,132.3,127.6,127.2$, 126.5, 124.8, 121.1, 88.1, 83.3, 51.3, 26.9, 23.4; MS (EI) m/z 184.1 ( $\mathrm{M}^{+}$(100.0), 152.1 (62.8), 141.1 (51.8), 115.1 (41.4)); Exact mass calc'd. for $\mathrm{C}_{13} \mathrm{H}_{12} \mathrm{O}$ : 184.0860 , found 184.0832.

(Cyclohex-1-enyl)-prop-2-yn-1-ol (41): $\mathrm{Pd}\left(\mathrm{PPh}_{3}\right)_{2} \mathrm{Cl}_{2}$ (175 mg, $\left.0.25 \mathrm{mmol}\right)$, copper (I) iodide (95 mg, $0.5 \mathrm{mmol})$, THF:DEA $(30 \mathrm{~mL})$, triflate $40(1.15 \mathrm{~g}, 5.0 \mathrm{mmol})$ in THF $(10 \mathrm{~mL})$, and propargyl alcohol $(0.29 \mathrm{~mL}, 5.5 \mathrm{mmol})$ were reacted together. Chromatography (petroleum ether/ethyl acetate, 4:1) afforded 41 as a viscous red oil (431 mg, 64\%). IR (neat); 3619 (s), 3438 (s), 2929 (s), 2871 (s), 1442 (s) cm ${ }^{-1} ;{ }^{1} \mathrm{H} \mathrm{NMR}\left(\mathrm{CDCl}_{3}, 500 \mathrm{MHz}\right): \delta 6.10$ (quintet, $J=2.1$ $\mathrm{Hz}, 1 \mathrm{H}), 4.35$ (s, 2H), $2.10-2.05(\mathrm{~m}, 4 \mathrm{H}), 1.63-1.54(\mathrm{~m}, 4 \mathrm{H}) ;{ }^{13} \mathrm{C} \mathrm{NMR}\left(\mathrm{CDCl}_{3}, 125 \mathrm{MHz}\right)$ : $\delta 135.5,120.1,87.6,84.5,51.6,29.1,25.6,22.2,21.4 ; \mathrm{MS}(\mathrm{EI}) \mathrm{m} / \mathrm{z} 136.1\left(\mathrm{M}^{+}(100.0), 107.0\right.$ (51.7), 91.1 (58.1), 79.1 (78.1)); Exact mass calc'd. for $\mathrm{C}_{9} \mathrm{H}_{12} \mathrm{O}$ : 136.0887, found 135.0886.

Carbometallation of Propargyl Alcohols: The appropriate vinyl propargyl alcohol was dissolved in either toluene or cyclohexane. Vinyl magnesium chloride in THF (3.2 eq) was added via syringe and the resulting solution refluxed for 16 hours. The reaction was cooled to $0{ }^{\circ} \mathrm{C}$, the appropriate electrophile added, and stirred for 30 minutes. The reaction was quenched with water $(50 \mathrm{~mL})$ or saturated aqueous ammonium chloride solution. The reaction mixture was extracted with diethyl ether $(2 \times 50 \mathrm{~mL})$ and the concentrated extract purified by silica gel chromatography with an appropriate petroleum ether/ethyl acetate solvent eluent. (More recent experiments have indicated yields are increased for these Grignard additions by addition of $10 \mathrm{~mol}$ percent of $\mathrm{CuI}$ and an equivalent of $\mathrm{MgCl}_{2}$ )

Tricyclo[6.2.1.0 $\left.{ }^{2,7}\right]$ undeca-2,4,-dien-4-methanol (9): Compound 8 (210 mg, $\left.1.42 \mathrm{mmol}\right)$ in toluene $(5 \mathrm{~mL})$, and vinyl magnesium chloride $(2.7 \mathrm{~mL}, 1.7 \mathrm{M}, 4.54 \mathrm{mmol})$ were reacted together and the reaction quenched with saturated ammonium chloride $(30 \mathrm{~mL})$. Chromatrography (petroleum ether/ethyl acetate 6:1) afforded 9 as an orange oil (105 $\mathrm{mg}, 42 \%)$. IR $\left(\mathrm{CDCl}_{3}\right) ; 3439$ (m), 2958 (s), 2867 (s), 1469 (m), $1371(\mathrm{~m}) \mathrm{cm}^{-1} ;{ }^{1} \mathrm{H}$ NMR $\left(\mathrm{CDCl}_{3}, 300\right.$ $\mathrm{MHz}): \delta 5.57(\mathrm{~s}, 1 \mathrm{H}), 5.55-5.53(\mathrm{~m}, 1 \mathrm{H}), 4.04(\mathrm{q}, J=12.3 \mathrm{~Hz}, 2 \mathrm{H}), 2.71(\mathrm{~s}, 1 \mathrm{H}), 2.15-2.02$ $(\mathrm{m}, 3 \mathrm{H}), 1.82-1.59(\mathrm{~m}, 4 \mathrm{H}), 1.47$ (br s, $1 \mathrm{H}), 1.42-1.30(\mathrm{~m}, 4 \mathrm{H}) ;{ }^{13} \mathrm{C} \mathrm{NMR}\left(\mathrm{CDCl}_{3}, 75 \mathrm{MHz}\right)$ : $\delta 153.4,137.8,120.0,110.4,66.1,44.4,44.2,42.1,39.5,31.0,27.2,26.5 ; \mathrm{MS}(\mathrm{EI}) \mathrm{m} / \mathrm{z} 176.1$ 
$\left(\mathrm{M}^{+}\right.$(26.0), 145.1 (48.3), 117.1 (87.1), 105.1 (96.9), 91.1 (100.0), 39.0 (60.7)); Exact mass calc'd. for $\mathrm{C}_{12} \mathrm{H}_{16} \mathrm{O}: 176.1183$, found 176.1164 .

1,11,11-Trimethyltricyclo[6.2.1.0 $\left.{ }^{2,7}\right]$ undeca-2,4,-dien-4-methanol (12): Compound 11 (467 $\mathrm{mg}, 2.46 \mathrm{mmol})$ in cyclohexane $(10 \mathrm{~mL})$, and vinyl magnesium chloride $(6.15 \mathrm{~mL}, 1.6 \mathrm{M}, 9.84$ mmol) were reacted together and the reaction quenched with saturated ammonium chloride (30 $\mathrm{mL}$ ). Chromatrography (petroleum ether/ethyl acetate 7:1) afforded 12 as an orange oil (391 mg, 73\%). IR (neat); 3355 (s), 2952 (s), 2869 (s), 1670 (m), 1448 (m), 1045 (m) cm ${ }^{-1}$; ${ }^{1} \mathrm{H}$ NMR $\left(\mathrm{C}_{6} \mathrm{D}_{6}, 200 \mathrm{MHz}\right): \delta 5.59(\mathrm{~s}, 1 \mathrm{H}), 5.52-5.49(\mathrm{~m}, 1 \mathrm{H}), 4.07(\mathrm{~s}, 2 \mathrm{H}), 2.55-2.39(\mathrm{~m}, 1 \mathrm{H}), 2.20-$ $2.01(\mathrm{~m}, 2 \mathrm{H}), 1.89-1.71(\mathrm{~m}, 3 \mathrm{H}), 1.31-1.13(\mathrm{~m}, 1 \mathrm{H}), 1.04(\mathrm{~s}, 3 \mathrm{H}), 0.96(\mathrm{~s}, 3 \mathrm{H}), 0.83(\mathrm{~s}, 3 \mathrm{H})$; ${ }^{13} \mathrm{C}$ NMR $\left(\mathrm{C}_{6} \mathrm{D}_{6}, 50 \mathrm{MHz}\right): \delta 154.3,137.5,118.0,109.8,65.5,50.9,50.4,48.1,45.5,32.3,30.5$, 27.7, 21.8, 20.7, 11.8; MS (EI) m/z 218.0 (M+ (17.3), 216.0 (20.9), 171.0 (34.8), 157.0 (83.0), 143.0 (100.0); Exact mass calc'd. for $\mathrm{C}_{15} \mathrm{H}_{22} \mathrm{O}: 218.1685$, found 218.1700 .

(6,6-Dimethylbicyclo[3.1.1]hept-2-en-2-yl)buta-1,3-dien-2-methanol (15): Compound (14) $(1.0 \mathrm{~g}, 5.3 \mathrm{mmol})$ in toluene $(10 \mathrm{~mL})$ and vinyl magnesium chloride $(10 \mathrm{~mL}, 1.7 \mathrm{M}, 16.96 \mathrm{mmol})$ were reacted together and the reaction quenched with saturated ammonium chloride $(30 \mathrm{~mL})$. Chromatrography (petroleum ether/ethyl acetate 7:1 afforded 15 as an orange oil (830 mg, 77\%). IR (neat); 3357 (s), 2990 (s), 2920 (s), 1461 (m), 1008 (m) cm ${ }^{-1} ;{ }^{1} \mathrm{H}$ NMR (CDCl $\left.3,300 \mathrm{MHz}\right)$ : $\delta 6.75$ (ddd, $J=0.9 \mathrm{~Hz}, 11.2 \mathrm{~Hz}, 17.9 \mathrm{~Hz}, 1 \mathrm{H}), 5.96(\mathrm{~s}, 1 \mathrm{H}), 5.54-5.57$ (m, 1H), $5.31(\mathrm{~d}, J=$ $17.9 \mathrm{~Hz}, 1 \mathrm{H}), 5.10(\mathrm{dt}, J=11.2 \mathrm{~Hz}, 1.4 \mathrm{~Hz}, 1 \mathrm{H}), 4.28(\mathrm{~s}, 2 \mathrm{H}), 2.34-2.40(\mathrm{~m}, 3 \mathrm{H}), 2.24(\mathrm{dt}, J=$ $1.4 \mathrm{~Hz}, 5.7 \mathrm{~Hz}, 1 \mathrm{H}), 2.05-2.11(\mathrm{~m}, 1 \mathrm{H}), 1.73($ br s, $1 \mathrm{H}), 1.27(\mathrm{~s}, 3 \mathrm{H}), 1.19$ (d, J=8.7 Hz, 1H), $0.85(\mathrm{~s}, 3 \mathrm{H}) ;{ }^{13} \mathrm{C} \mathrm{NMR}\left(\mathrm{CDCl}_{3}, 75 \mathrm{MHz}\right): \delta 144.8$ 135.3, 133.6, 131.5, 125.5, 114.4, 65.1, 46.9, 40.7, 38.2, 32.4, 31.9, 26.6, 21.5; MS (EI) m/z $204.2\left(\mathrm{M}^{+}\right.$(15.2), 161.1 (24.0), 143.1 (38.8), 129.1 (52.8), 117.1 (43.9), 105.1 (100.0), 91.1 (61.3)); Exact mass calc'd. for $\mathrm{C}_{14} \mathrm{H}_{20} \mathrm{O}$ : 204.1501, found 204.1487.

(Bicyclo[3.2.1]oct-2-en-2-yl)buta-1,3-dien-2-methanol (18): Compound 17 (1.0 g, 6.17 mmol) in toluene $(15 \mathrm{~mL})$ and vinyl magnesium chloride $(11.6 \mathrm{~mL}, 1.7 \mathrm{M}, 19.7 \mathrm{mmol})$ were reacted together and the reaction quenched with saturated ammonium chloride $(30 \mathrm{~mL})$. Chromatography (petroleum ether/ethyl acetate 8:1) afforded 18 as a yellow oil (750 $\mathrm{mg}, 64 \%)$. IR (neat); 3329 (s), 2936 (s), 2865 (s), 1442 (m), 1088 (m) cm ${ }^{-1} ;{ }^{1} \mathrm{H}$ NMR (CDCl, $\left.300 \mathrm{MHz}\right)$ : $\delta 6.74(\mathrm{dd}, J=17.9 \mathrm{~Hz}, 11.3 \mathrm{~Hz}, 1 \mathrm{H}), 5.97(\mathrm{~m}, 1 \mathrm{H}), 5.34(\mathrm{~m}, 1 \mathrm{H}), 5.31(\mathrm{~d}, J=17.8 \mathrm{~Hz}, 1 \mathrm{H})$, $5.09(\mathrm{~d}, J=11.2 \mathrm{~Hz}, 1 \mathrm{H}), 4.30(\mathrm{~s}, 2 \mathrm{H}), 2.47-2.31(\mathrm{~m}, 3 \mathrm{H}), 2.01-1.38(\mathrm{~m}, 8 \mathrm{H}) ;{ }^{13} \mathrm{C}$ NMR $\left(\mathrm{CDCl}_{3}, 75 \mathrm{MHz}\right): \delta 142.5,135.5,133.5,132.3,126.1,114.2,65.2,41.6,37.8,35.6,35.2,33.1$, 31.1; MS (EI) m/z 190.1 (M+ (20.8), 159.1 (23.0), 129.1 (29.8), 105.1 (29.0), 91.1 (100.0), 79.1 (43.4)); Exact mass calc'd. for $\mathrm{C}_{13} \mathrm{H}_{18} \mathrm{O}$ : 190.1349, found 190.1341.

(Bicyclo[3.2.1]oct-2-en-2-yl)buta-1,3-dien-2-methoxymethyl (22): Compound 18 (680 mg, 3.6 mmol) was dissolved in dry THF $(20 \mathrm{~mL})$. $\mathrm{KO}^{\mathrm{t}} \mathrm{Bu}$ in THF $(17.9 \mathrm{~mL}, 1 \mathrm{M}, 18.0 \mathrm{mmol})$ was added via syringe and stirred for 10 minutes. MeI $(1.98 \mathrm{~mL}, 18.0 \mathrm{mmol})$ was added via syringe and the reaction stirred at room temperature for 3 hours. The reaction was quenched with water $(50 \mathrm{~mL})$ and extracted with diethyl ether $(3 \times 30 \mathrm{~mL})$ to afford 22 as a yellow oil $(728 \mathrm{mg}, 99 \%)$. IR (neat); 2943 (s), 2865 (m), 1448 (w), $1094(\mathrm{~m}) \mathrm{cm}^{-1} ;{ }^{1} \mathrm{H} \mathrm{NMR}\left(\mathrm{CDCl}_{3}, 300 \mathrm{MHz}\right): \delta 6.75$ (dd, $J$ $=17.8 \mathrm{~Hz}, 11.2 \mathrm{~Hz}, 1 \mathrm{H}), 5.93(\mathrm{~m}, 1 \mathrm{H}), 5.34(\mathrm{~m}, 1 \mathrm{H}), 5.31(\mathrm{~d}, \mathrm{~J}=18.1 \mathrm{~Hz}, 1 \mathrm{H}), 5.06(\mathrm{~d}, J=11.2$ $\mathrm{Hz}, 1 \mathrm{H}), 4.06(\mathrm{~s}, 2 \mathrm{H}), 3.31(\mathrm{~s}, 3 \mathrm{H}), 2.47-2.25(\mathrm{~m}, 3 \mathrm{H}), 1.95-1.59(\mathrm{~m}, 5 \mathrm{H}), 1.52-1.38(\mathrm{~m}$, 
$2 \mathrm{H}) ;{ }^{13} \mathrm{C} \mathrm{NMR}\left(\mathrm{CDCl}_{3}, 75 \mathrm{MHz}\right): \delta 142.5,133.6,132.7,131.4,125.9,114.4,75.0,58.2,41.5$, 39.8, 35.6, 35.2, 32.7, 29.2; MS (EI) m/z 204.2 (M+ (27.8), 159.1 (22.0), 129.1 (41.6), 104.1 (45.1), 91.1 (100.0), 45.0 (66.3)); Exact mass calc'd. for $\mathrm{C}_{14} \mathrm{H}_{20} \mathrm{O}: 204.1520$, found 204.1526.

\section{7,7-Dimethylbicyclo[3.1.1] hept-2,3-benzo-3'-carbaldehyde (23):}

Compound $15(150 \mathrm{mg}, 0.74 \mathrm{mmol})$ was dissolved in toluene $(10 \mathrm{~mL})$ in a sealed tube heated at $150{ }^{\circ} \mathrm{C}$ for $16 \mathrm{~h}$. The crude product was added to toluene $(20 \mathrm{~mL})$, DDQ (500 $\left.\mathrm{mg}, 1.85 \mathrm{mmol}\right)$ was added and the reaction mixture refluxed for 3 hours. The DDQ was removed by filtration and the toluene removed with a rotary evaporator under reduced pressure. Chromatography (petroleum ether/ethyl acetate 20:1) afforded 23 as a yellow oil (82 $\mathrm{mg}, 56 \%$ ).IR (neat); 2946 (m), 2840 (m), 1609 (m), 1444 (s), 1248 (s) cm ${ }^{-1} ;{ }^{1} \mathrm{H}$ NMR $\left(\mathrm{CDCl}_{3}, 300 \mathrm{MHz}\right): \delta 9.90(\mathrm{~s}, 1 \mathrm{H})$, $7.62(\mathrm{~d}, J=7.7 \mathrm{~Hz}, 1 \mathrm{H}), 7.41(\mathrm{~s}, 1 \mathrm{H}), 7.25(\mathrm{~d}, J=7.2 \mathrm{~Hz}, 1 \mathrm{H}), 3.02(\mathrm{~s}, 2 \mathrm{H}), 2.82(\mathrm{t}, J=5.5 \mathrm{~Hz}$, $1 \mathrm{H}), 2.69-2.62(\mathrm{~m}, 1 \mathrm{H}), 2.31-2.27(\mathrm{~m}, 1 \mathrm{H}), 1.38(\mathrm{~s}, 3 \mathrm{H}), 1.20(\mathrm{~d}, J=9.6 \mathrm{~Hz}, 1 \mathrm{H}), 0.60(\mathrm{~s}$, $3 \mathrm{H}) ;{ }^{13} \mathrm{C} \mathrm{NMR}\left(\mathrm{CDCl}_{3}, 75 \mathrm{MHz}\right): \delta 192.8,148.2,143.5,134.2,128.9,128.8,126.7,48.0,40.5$,

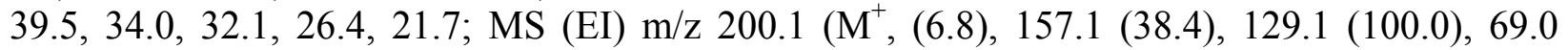
(28.7); Exact mass calc'd. for $\mathrm{C}_{14} \mathrm{H}_{16} \mathrm{O}$ : 200.1202, found 200.1201 .

7,7-Dimethylbicyclo[3.1.1]hept-2,3-benzo-3'-hydroxymethyl (21): Vinyl magnesium bromide (Acros Organics, 15\% wt in THF, $2.6 \mathrm{~mL}, 4.34 \mathrm{mmol}$ ) was added dropwise to a solution of 7,7Dimethylbicyclo[3.1.1]hept-2-en-2-yl)-prop-2-yn-1-ol (14) (191 mg, $1.08 \mathrm{mmol})$ in toluene:THF $(5: 1,13 \mathrm{~mL})$. The brown reaction mixture stirred at reflux for $16 \mathrm{~h}$. The reaction was quenched with saturated ammonium chloride solution $(10 \mathrm{~mL})$ at $0^{\circ} \mathrm{C}$ and the mixture stirred for $10 \mathrm{~min}$. Dichloromethane was added followed by water and the aqueous phase was extracted with dichloromethane (3x). The combined organic phases was washed with $\mathrm{H}_{2} \mathrm{O}(2 \mathrm{x})$ and brine, dried with $\mathrm{MgSO}_{4}$ and concentrated. Chromatography (petroleum ether/diethyl ether, 3:1) afforded product as a clear oil (115 mg, 53\%). IR (neat) v 3336.6, 2980.6, 2916.2, 2867.7, 2833.5, 1721.4, 1622.1, 1495.4, 1025.1 cm $\mathrm{cm}^{-1}{ }^{1} \mathrm{H}$ NMR $\left(500 \mathrm{MHz}, \mathrm{CDCl}_{3}\right) \delta 7.11-7.07(\mathrm{~m}, 2 \mathrm{H}) 6.90(\mathrm{~s}, 1 \mathrm{H}) 4.60$ (s, 2H) $2.94(\mathrm{bs}, 2 \mathrm{H}) 2.71(\mathrm{t}, 5.5 \mathrm{~Hz}, 1 \mathrm{H}) 2.60(\mathrm{dt}, 9.3,5.9 \mathrm{~Hz}, 1 \mathrm{H}) 2.25(\mathrm{~m}, 1 \mathrm{H}) 1.35(\mathrm{~s}, 3 \mathrm{H})$ $1.23(\mathrm{bs}, 1 \mathrm{H}) 1.21(\mathrm{~d}, 9.3 \mathrm{~Hz}, 1 \mathrm{H}) 0.61(\mathrm{~s}, 3 \mathrm{H}) ;{ }^{13} \mathrm{C} \mathrm{NMR}\left(125 \mathrm{MHz}, \mathrm{CDCl}_{3}\right) \delta 147.1,137.3$, 134.4, 127.8, 124.7, 124.5, 62.4, 47.7, 40.4, 39.0, 32.6, 31.8, 26.0, 21.2; MS (EI) $\mathrm{m} / z 202.1\left(\mathrm{M}^{+}\right.$, 8.5) 159.1 (14.2) 129.1 (100) 115.1 (7.2); HRMS calcd for $\mathrm{C}_{14} \mathrm{H}_{18} \mathrm{O} 202.1356\left(\mathrm{M}^{+}\right)$, found 202.1353 .

Bicyclo[3.2.1]oct-2,3-benzo-3'-carbaldehyde (24): Compound 25 (90.0 mg, $0.44 \mathrm{mmol})$ was dissolved in toluene $(30 \mathrm{~mL})$. DDQ $(245 \mathrm{mg}, 0.88 \mathrm{mmol})$ was added and the reaction was refluxed and stirred for 3 hours. The reaction was quenched with water $(30 \mathrm{~mL})$ and extracted with diethyl ether (3 x $20 \mathrm{~mL})$. Chromatrography (petroleum ether/ethyl acetate 19:1) afforded 24 as a yellow oil (52 mg, 64\%). IR ( $\left.\mathrm{CDCl}_{3}\right) ; 2949(\mathrm{~m}), 2872(\mathrm{w}), 1693(\mathrm{~s}), 1609$ (m), $1223(\mathrm{w})$ $\mathrm{cm}^{-1}$; ${ }^{1} \mathrm{H} \mathrm{NMR}\left(\mathrm{CDCl}_{3}, 500 \mathrm{MHz}\right): \delta 9.90(\mathrm{~s}, 1 \mathrm{H}), 7.57(\mathrm{dd}, J=7.8 \mathrm{~Hz}, 1.8 \mathrm{~Hz}, 1 \mathrm{H}), 7.49(\mathrm{~d}, J=$ $7.17(\mathrm{~d}, J=7.8 \mathrm{~Hz}, 1 \mathrm{H}), 3.13-3.07(\mathrm{~m}, 2 \mathrm{H}), 2.67(\mathrm{~d}, J=17.8 \mathrm{~Hz}, 1 \mathrm{H}), 2.57-2.54(\mathrm{~m}, 1 \mathrm{H})$, $1.98-1.89(\mathrm{~m}, 2 \mathrm{H}), 1.80-1.72(\mathrm{~m}, 3 \mathrm{H}), 1.48-1.44(\mathrm{~m}, 1 \mathrm{H}) ;{ }^{13} \mathrm{C} \mathrm{NMR}\left(\mathrm{CDCl}_{3}, 125 \mathrm{MHz}\right)$ : $\delta 192.3,146.3,142.8,134.3,130.1,128.2,127.4,41.1,40.0,36.2,35.3,33.3,29.7 ; \mathrm{MS}(\mathrm{EI}) \mathrm{m} / \mathrm{z}$ $186.1{\left(\mathrm{M}^{+}\right.}^{(42.4),} 162.0$ (9.0), 143.0 (7.2), 129.1 (100.0), 115.1 (20.8); Exact mass calc'd. for $\mathrm{C}_{13} \mathrm{H}_{14} \mathrm{O}: 186.1012$, found 186.0979 . 
1,11,11,3'-Tetramethyltricyclo[6.2.1.0 $\left.{ }^{2,7}\right]$ undeca-2,4,-dien-4-methanol $\quad$ (26): Propargyl alcohol 11 (465 mg, $2.45 \mathrm{mmol})$ in cyclohexane $(5 \mathrm{~mL})$, vinyl magnesium chloride $(4.6 \mathrm{~mL}, 1.7$ $\mathrm{M}, 7.84 \mathrm{mmol})$, and $\mathrm{MeI}(1.5 \mathrm{~mL}, 24.5 \mathrm{mmol})$ were reacted together. Chromatography (petroleum ether/ethyl acetate, 6:1) afforded 26 as a yellow oil (520 mg, 92\%). IR (neat) 3370 (s), $2954(\mathrm{~s}), 2872(\mathrm{~s}), 1472(\mathrm{~m}), 1387(\mathrm{~m}) \mathrm{cm}^{-1} ;{ }^{1} \mathrm{H}$ NMR $\left(\mathrm{CDCl}_{3}, 300 \mathrm{MHz}\right): \delta 5.65(\mathrm{t}, J=3.9$ $\mathrm{Hz}, 1 \mathrm{H}), 4.22(\mathrm{~d}, J=12.3 \mathrm{~Hz}, 1 \mathrm{H}), 4.02(\mathrm{~d}, J=12.3 \mathrm{~Hz}, 1 \mathrm{H}), 2.37-2.24(\mathrm{~m}, 1 \mathrm{H}), 2.07-1.99$ $(\mathrm{m}, 1 \mathrm{H}), 1.85(\mathrm{~s}, 3 \mathrm{H}), 1.59-1.49(\mathrm{~m}, 3 \mathrm{H}), 1.27-1.21(\mathrm{~m}, 2 \mathrm{H}), 1.17(\mathrm{~s}, 3 \mathrm{H}), 1.04(\mathrm{~s}, 3 \mathrm{H}), 0.99-$ $0.88(\mathrm{~m}, 1 \mathrm{H}), 0.83(\mathrm{~s}, 3 \mathrm{H}) ;{ }^{13} \mathrm{C} \mathrm{NMR}\left(\mathrm{CDCl}_{3}, 75 \mathrm{MHz}\right): \delta 146.4,140.2,122.9,118.5,65.1,53.0$, 52.0, 48.1, 47.8, 34.5, 31.1, 28.2, 22.9, 21.5, 16.5, 13.0; MS (EI) m/z 232.2 (M (53.1), 145.1 (87.7), 133.1 (100.0), 105.1 (67.4), 41.0 (71.9)); Exact mass calc'd. for $\mathrm{C}_{16} \mathrm{H}_{24} \mathrm{O}: 232.1804$, found 232.1781 .

4,14,14-Trimethyl-7-boryl-(1-hydroxy)-8-oxa-tetracyclo $\left[9.2 .1 \cdot 0 .{ }^{5,13} 0^{6,10}\right]$ dodeca-5,10- diene (28): Propargyl alcohol 11 (430 mg, $2.26 \mathrm{mmol}$ ) and magnesium chloride (323 mg, $3.39 \mathrm{mmol})$ were dissolved in dry cyclohexane $(5 \mathrm{~mL})$. Vinyl magnesium chloride $(4.3 \mathrm{~mL}, 1.7 \mathrm{M}, 7.23$ mmol) was added via syringe at room temperature and the resulting red solution refluxed for 14 hours. The solution was cooled to $0{ }^{\circ} \mathrm{C}$ and quenched with trimethyl borate $(0.76 \mathrm{~mL}, 6.78$ $\mathrm{mmol})$ and stirred for 1 hour. The reaction was quenched with water $(30 \mathrm{~mL})$ and extracted with diethyl ether (3 x $20 \mathrm{~mL})$. Chromatography (petroleum ether/ethyl acetate, 15:1) afforded 28 as white oily solid (303 mg, 55\%). IR (neat); 3612 (m), 3296 (m), 2955 (s), 1667 (s), 1402 (s), $1255(\mathrm{~m}) \mathrm{cm}^{-1} ;{ }^{1} \mathrm{H} \mathrm{NMR}\left(\mathrm{CDCl}_{3}, 300 \mathrm{MHz}\right): \delta 5.22$ (br s, 1H), $5.11(\mathrm{~s}, 1 \mathrm{H}), 4.61(\mathrm{~s}, 2 \mathrm{H}), 2.46-$ $2.37(\mathrm{~m}, 1 \mathrm{H}), 2.23-2.09(\mathrm{~m}, 1 \mathrm{H}), 1.96-1.85(\mathrm{~m}, 1 \mathrm{H}), 1.72-1.63(\mathrm{~m}, 2 \mathrm{H}), 1.56-1.43(\mathrm{~m}$, $1 \mathrm{H}), 1.32-1.20(\mathrm{~m}, 1 \mathrm{H}), 1.18(\mathrm{~s}, 3 \mathrm{H}), 0.99(\mathrm{~s}, 3 \mathrm{H}), 0.89(\mathrm{~s}, 3 \mathrm{H}), 0.83-0.76(\mathrm{~m}, 1 \mathrm{H}) ;{ }^{13} \mathrm{C} \mathrm{NMR}$ $\left(\mathrm{CDCl}_{3}, 75 \mathrm{MHz}\right): \delta 167.9,142.4,110.8,70.8,53.7,52.7,48.3,48.2,32.9,30.6,28.1,22.6,21.4$, 14.0; MS (EI) m/z 244.2 (M+, (11.6), 174.1 (68.8), 162.0 (36.3), 143.0 (31.8), 131.1 (100.0), 100.0 (65.5), 69.0 (78.7)); Exact mass calc'd. for $\mathrm{C}_{13} \mathrm{H}_{21} \mathrm{BO}_{2}: 244.1642$, found 244.1650.

\section{1,7,7-Trimethyl-2,3-benzo-(5,6-(1-hydroxybora2-2oxacyclopentan)-bicyclo[2.2.1]heptane}

(30): DDQ (70.4 mg, $0.309 \mathrm{mmol}, 1.1$ equiv) was added to a solution of 28 (68.8 $\mathrm{mg}, 0.281$ mmol) in toluene $(1.87 \mathrm{~mL})$. The mixture was refluxed for $4 \mathrm{hrs}$. The mixture was diluted with water and the aqueous phase was extracted with diethyl ether $(3 \times 10 \mathrm{~mL})$. The combined organic layers were washed with water $(1 \times 10 \mathrm{~mL})$, brine $(1 \times 10 \mathrm{~mL})$ and dried over $\mathrm{MgSO}_{4}$. Chromatography (petroleum ether/ $\left.\mathrm{Et}_{2} \mathrm{O}, 19: 1\right)$ afforded $30(32.6 \mathrm{mg}, 48 \%)$ as a white solid (mp 256-258). IR (neat) 3358, 3050, 2953, 2872, 1709, 1604, 1490, 1470, 1445, 1430, 1405, 1376, 1278, 1196, 1020, 985, $795 \mathrm{~cm}^{-1} .{ }^{1} \mathrm{H}$ NMR $\left(\mathrm{CDCl}_{3}, 500 \mathrm{MHz}\right): \delta 7.21(\mathrm{~d}, J=7.4,1 \mathrm{H}), 7.01(\mathrm{~d}, J$ $=7.4,1 \mathrm{H}), 5.39(\mathrm{~s}, 1 \mathrm{H}) 5.10-5.03(\mathrm{~m}, 2 \mathrm{H}) 2.81(\mathrm{~d}, J=3.9,1 \mathrm{H}) 2.09-2.03(\mathrm{~m}, 1 \mathrm{H}) 1.86-1.81(\mathrm{~m}$, 1H) $1.49(\mathrm{~s}, 3 \mathrm{H}) 1.17-1.12(\mathrm{~m}, 1 \mathrm{H}) 1.08-1.03(\mathrm{~m}, 1 \mathrm{H}) 0.95(\mathrm{~s}, 3 \mathrm{H}) 0.53(\mathrm{~s}, 3 \mathrm{H}) .{ }^{13} \mathrm{C} \mathrm{NMR}$ $\left(\mathrm{CDCl}_{3}, 126 \mathrm{MHz}\right): \delta 155.3,151.6,146.9,123.8,117.5,71.3,57.6,54.8,53.0,33.2,33.1,26.3$, 20.1, 19.3, 12.9. Exact mass calc'd. for $\mathrm{C}_{15} \mathrm{H}_{19} \mathrm{BO}$ : 242. 1478, found 242.1478.

\section{1,11,11-Trimethyltricyclo $\left[6.2 .1 .0^{2,7}\right]$ undeca-2,4,-dien-3-iodo-4-hydroxymethanol}

(29): Magnesium chloride $(10.33 \mathrm{~g}, 108.5 \mathrm{mmol})$ was added to a solution of propargyl alcohol 11 $(9.38 \mathrm{~g}, 49.30 \mathrm{mmol})$ in cyclohexane $(100 \mathrm{~mL})$, and a solution of vinyl magnesium chloride in THF (1.6 M, $108 \mathrm{~mL}, 172.5 \mathrm{mmol})$ was then added. The mixture was heated at reflux overnight. The reaction was then cooled to $0{ }^{\circ} \mathrm{C}$ and a solution of $\mathrm{I}_{2}(37.54 \mathrm{~g}, 147.9 \mathrm{mmol})$ in $\mathrm{THF}(140$ $\mathrm{mL}$ ) was added until the dark brown color of $\mathrm{I}_{2}$ persisted. After stirring at $0{ }^{\circ} \mathrm{C}$ for 2 hours, the 
reaction was quenched via the addition of $1 \mathrm{M} \mathrm{Na}_{2} \mathrm{~S}_{2} \mathrm{O}_{3}(200 \mathrm{~mL})$ and extracted with ether $(3 \mathrm{x}$ $300 \mathrm{~mL})$. The organics were combined, dried $\left(\mathrm{MgSO}_{4}\right)$, filtered, and concentrated. Column chromatography (10:1, petroleum ether:ethyl acetate) afforded 29 as a yellow oil that solidified upon refrigeration $(13.77 \mathrm{~g}, 81 \%)$. Yields varied from $70-80 \%$. mp $<20{ }^{\circ} \mathrm{C}$. ${ }^{1} \mathrm{H}$ NMR $(500$ $\left.\mathrm{MHz}, \mathrm{CDCl}_{3}\right) \delta$ 5.68-5.70 (m, 1H), 4.28-4.32 (m, 1H), $4.14(\mathrm{ddd}, J=13.6,1.4$ and $1.4 \mathrm{~Hz}, 1 \mathrm{H})$, $2.46(\mathrm{dd}, J=18.9$ and $8.2 \mathrm{~Hz}, 1 \mathrm{H}), 1.95-2.04(\mathrm{~m}, 1 \mathrm{H}), 1.73-1.79(\mathrm{~m}, 1 \mathrm{H}), 1.58-1.68(\mathrm{~m}, 2 \mathrm{H})$, 1.48-1.54 (m, 1H), 1.36-1.42 (m, 1H), $1.29(\mathrm{~s}, 3 \mathrm{H}), 1.24(\mathrm{~d}, J=3.8 \mathrm{~Hz}, 1 \mathrm{H}), 1.00-1.05(\mathrm{~m}, 1 \mathrm{H})$, $0.97(\mathrm{~s}, 3 \mathrm{H}), 0.68(\mathrm{~s}, 3 \mathrm{H}) ;{ }^{13} \mathrm{C}$ NMR $\left(125 \mathrm{MHz}, \mathrm{CDCl}_{3}\right) \delta 155.8,138.6,123.4,79.1,69.1,55.7$, $53.2,52.5,48.4,34.0,31.2,28.0,22.9,21.7,16.8$; IR (neat): $v=3329,2956,2930,2870,1625$, 1388, 1448, 1304, 1127, 1053, 987, $786 \mathrm{~cm}^{-1}$; MS (EI) m/z $344\left(\mathrm{M}^{+}\right)$(100), 257 (16), 199 (18), 118 (41), 91 (34), 69 (26), 41 (45); HRMS calculated for $\mathrm{C}_{15} \mathrm{H}_{21} \mathrm{OI}\left(\mathrm{M}^{+}\right.$) 344.0637, found 344.0652 .

1,7,7-Trimethylbicyclo[2.2.1]hept-2,3-benzo-2'-iodophenol (33): Aldehyde 31 (2.00 g, 5.88 mmol) was dissolved in $\mathrm{CH}_{2} \mathrm{Cl}_{2}(100 \mathrm{~mL})$ and cooled to $-8{ }^{\circ} \mathrm{C}$. $m$-Chloroperoxybenzoic acid (purified using a phosphate buffer of $\mathrm{pH} 7.4)$ (1.52 $\mathrm{g}, 8.82 \mathrm{mmol}$ ) was added and the mixture was permitted to warm to $0{ }^{\circ} \mathrm{C}$ over a period of 30 minutes, and then held at that temperature for a further 60 minutes. After removal of the solvent, the solid was dissolved in 30:1 petroleum ether:ethyl acetate and filtered through a pad of silica gel, which was washed with the same solvent until no further product eluted. After removal of the solvent, the crude formate (mixed with the phenol) was dissolved in $\mathrm{MeOH}(35 \mathrm{~mL})$ and $\mathrm{TsOH}(0.2 \mathrm{~g})$ was added. After stirring at $\mathrm{rt}$ for one hour, the mixture was diluted with water $(30 \mathrm{~mL})$ and extracted with ether $(3 \times 60 \mathrm{~mL})$. The organics were combined, dried $\left(\mathrm{MgSO}_{4}\right)$, filtered, and concentrated. Column chromatography (50:1, petroleum ether:ethyl acetate) afforded 33 as a gummy solid (1.10 g, 57\%). ${ }^{1} \mathrm{H}$ NMR $\left(500 \mathrm{MHz}, \mathrm{C}_{6} \mathrm{D}_{6}\right) \delta 6.91(\mathrm{~d}, J=7.7 \mathrm{~Hz}, 1 \mathrm{H}), 6.73(\mathrm{~d}, J=7.7 \mathrm{~Hz}, 1 \mathrm{H}), 2.66(\mathrm{~d}, J$ $=3.8 \mathrm{~Hz}, 1 \mathrm{H}), 1.98-2.04(\mathrm{~m}, 1 \mathrm{H}), 1.73(\mathrm{ddd}, J=12.4,10.0$ and $3.9 \mathrm{~Hz}, 1 \mathrm{H}), 1.43(\mathrm{~s}, 3 \mathrm{H}), 1.24$ (ddd, $J=12.8,9.5$ and $3.3 \mathrm{~Hz}, 1 \mathrm{H}$ ), 1.04 (ddd, $J=12.7,9.2$ and $3.9 \mathrm{~Hz}, 1 \mathrm{H}), 0.90$ (s, $3 \mathrm{H}), 0.57$ $(\mathrm{s}, 3 \mathrm{H}) ;{ }^{13} \mathrm{C}$ NMR $\left(125 \mathrm{MHz}, \mathrm{C}_{6} \mathrm{D}_{6}\right) \delta 151.9,150.7,142.6,122.2,111.1,82.4,57.5,57.4,52.3$, 32.5, 26.6, 20.1, 19.4, 14.6; IR (neat): $v=3475,3055,2956,2871,1560,1433,1226,1177$, 1062, 908, 820, $780 \mathrm{~cm}^{-1}$; MS (EI) $\mathrm{m} / z 328\left(\mathrm{M}^{+}\right)$(18), 285 (15), 186 (4), 158 (46), 59 (34), 31 (100); HRMS calculated for $\mathrm{C}_{25} \mathrm{H}_{30} \mathrm{I}_{2}\left(\mathrm{M}^{+}\right)$328.0324, found 328.0339.

1,11,11-Trimethylbicyclo[2.2.1]hept-2,3-benzo-2'-iodo-3'-carbaldehyde (31): Compound 29 (5-Iodo-4,9,9-trimethyl-1,2,3,4,8,8a-hexahydro-1,4-methano-naphthalen-6-yl)-methanol (0.59 g, $1.71 \mathrm{mmol})$ was dissolved in toluene $(65 \mathrm{~mL})$ and $\mathrm{MnO}_{2}(1.49 \mathrm{~g}, 17.14 \mathrm{mmol})$ was added. The reaction was heated at reflux for two days and then filtered through a silica pad using ether as eluent. Column chromatography (30:1, petroleum ether:ethyl acetate) afforded 31 as a yellow solid (0.43 g, 73\%). mp = 75.5-77.5 ${ }^{\circ} \mathrm{C} ;{ }^{1} \mathrm{H} \mathrm{NMR}\left(500 \mathrm{MHz}, \mathrm{CDCl}_{3}\right) \delta 10.24(\mathrm{~s}, 1 \mathrm{H}), 7.66(\mathrm{~d}$, $J=7.4 \mathrm{~Hz}, 1 \mathrm{H}), 7.16(\mathrm{~d}, J=7.4 \mathrm{~Hz}, 1 \mathrm{H}), 2.81(\mathrm{~d}, J=3.9 \mathrm{~Hz}, 1 \mathrm{H}), 2.10-2.16(\mathrm{~m}, 1 \mathrm{H}), 1.83$ (ddd, $J=13.8,10.2$ and $4.0 \mathrm{~Hz}, 1 \mathrm{H}), 1.56(\mathrm{~s}, 3 \mathrm{H}), 1.34$ (ddd, $J=12.8,9.3$ and $4.0 \mathrm{~Hz}, 1 \mathrm{H}), 1.09$ (ddd, $J=12.8,9.3$ and $4.0 \mathrm{~Hz}, 1 \mathrm{H}), 0.96(\mathrm{~s}, 3 \mathrm{H}), 0.60(\mathrm{~s}, 3 \mathrm{H}) ;{ }^{13} \mathrm{C} \mathrm{NMR}\left(125 \mathrm{MHz}, \mathrm{CDCl}_{3}\right) \delta 197.1$, $157.8,151.4,133.7,129.6,121.7,94.8,58.0,57.6,53.7,32.2,26.2,20.3,19.4,15.5$; IR (neat): $v$ $=3054,2987,2685,1684,1422,1258 \mathrm{~cm}^{-1}$; MS (EI) m/z $584\left(\mathrm{M}^{+}\right)$(51), 527 (10); HRMS calculated for $\mathrm{C}_{15} \mathrm{H}_{17} \mathrm{OI}\left(\mathrm{M}^{+}\right)$340.0313, found 340.0302 . 
1,11,11-Trimethylbicyclo[2.2.1]hept-2,3-benzo-2'-iodo-3'-carboxylic acid (32): Compound 31 (500 mg, $1.47 \mathrm{mmol})$ was dissolved in an aqueous solution of $t-\mathrm{BuOH}(50 \mathrm{~mL}, 5: 2)$. $\mathrm{NaH}_{2} \mathrm{PO}_{4}(706 \mathrm{mg}, 5.88 \mathrm{mmol})$ and 2-methyl butene $(1.09 \mathrm{~mL}, 10.29 \mathrm{mmol})$ were added and the solution allowed to stir for 10 minutes at room temperature. After addition of $\mathrm{NaClO}_{2}(798 \mathrm{mg}$, $8.82 \mathrm{mmol}$ ) the solution was stirred for 3 hours at room temperature. $t$-BuOH was removed on the rotary evaporator as the benzene azeotrope $(3 \times 20 \mathrm{~mL})$. The resulting white solid was dried under vacuum over night. Chromatography (petroleum ether/ethyl acetate, 19:1) afforded 32 as a yellow oil (520 mg, 98\%). ${ }^{1} \mathrm{H} \mathrm{NMR}\left(\mathrm{CDCl}_{3}, 300 \mathrm{MHz}\right): \delta 7.34(\mathrm{~d}, J=7.3 \mathrm{~Hz}, 1 \mathrm{H}), 7.04(\mathrm{~d}, J=$ $7.3 \mathrm{~Hz}, 1 \mathrm{H}), 2.71(\mathrm{~d}, J=3.7 \mathrm{~Hz}, 1 \mathrm{H}), 2.03(\mathrm{~m}, 1 \mathrm{H}), 1.72(\mathrm{dt}, J=4.0 \mathrm{~Hz}, 9.5 \mathrm{~Hz}, 1 \mathrm{H}), 1.47$ (s, $3 \mathrm{H}), 1.25(\mathrm{dt}, J=3.7 \mathrm{~Hz}, 9.2 \mathrm{~Hz}, 1 \mathrm{H}), 1.02(\mathrm{dt}, J=3.9 \mathrm{~Hz}, 12.5 \mathrm{~Hz}, 1 \mathrm{H}), 0.89(\mathrm{~s}, 3 \mathrm{H}), 0.52(\mathrm{~s}$, $3 \mathrm{H}) ;{ }^{13} \mathrm{C} \mathrm{NMR}\left(\mathrm{CDCl}_{3}, 75 \mathrm{MHz}\right): \delta 171.2,152.6,151.3,139.1,127.4,121.6,86.9,57.9,57.7$, 53.0, 32.6, 26.8, 20.7, 19.8, 16.0; MS (EI) m/z $356.0\left(\mathrm{M}^{+}\right.$, (100.0), 328.0 (33.6), 295.0 (25.7), 201.1 (32.5), 186.1 (93.2)); Exact mass calc'd. for $\mathrm{C}_{15} \mathrm{H}_{17} \mathrm{IO}_{2}: 356.0286$, found 356.0273.

5-Methoxy-3,4-dihydronaphthalen-1-yl)buta-1,3-dien-2-methanol (36a): Compound 35 (510 $\mathrm{mg}, 2.52 \mathrm{mmol})$ in toluene $(20 \mathrm{~mL})$ and vinyl magnesium chloride $(4.9 \mathrm{~mL}, 1.7 \mathrm{M}, 8.1 \mathrm{mmol})$ were reacted together and the reaction quenched with saturated ammonium chloride $(30 \mathrm{~mL})$. Chromatography (petroleum ether/ethyl acetate, 3:1) afforded 36a as a yellow oil (433 mg, 71\%). IR (neat); 3396 (s), 2934 (s), 2834 (s), 1567 (s), 1251 (s), 1042 (s) cm ${ }^{-1} ;{ }^{1} \mathrm{H} \mathrm{NMR}\left(\mathrm{CDCl}_{3}\right.$, $300 \mathrm{MHz}): \delta 7.06(\mathrm{~d}, J=8.4 \mathrm{~Hz}, 1 \mathrm{H}), 6.77-6.64(\mathrm{~m}, 3 \mathrm{H}), 6.45(\mathrm{~s}, 1 \mathrm{H}), 5.83(\mathrm{t}, J=4.0 \mathrm{~Hz}, 1 \mathrm{H})$, $5.32(\mathrm{t}, J=18.0 \mathrm{~Hz}, 1 \mathrm{H}), 5.10(\mathrm{~d}, J=11.3 \mathrm{~Hz}, 1 \mathrm{H}), 4.45(\mathrm{~s}, 2 \mathrm{H}), 3.77(\mathrm{~s}, 3 \mathrm{H}), 2.75(\mathrm{t}, J=7.7 \mathrm{~Hz}$, 2H), 2.56 (br s, 1H), $2.38-2.32(\mathrm{~m}, 1 \mathrm{H}) ;{ }^{13} \mathrm{C} \mathrm{NMR}\left(\mathrm{CDCl}_{3}, 125 \mathrm{MHz}\right): \delta 159.1,139.0,138.2$, 133.5, 128.7, 128.3, 127.8, 125.9, 114.5, 114.2, 111.3, 64.3, 55.6, 28.8, 23.6; MS (EI) m/z 242.1 $\left(\mathrm{M}^{+}\right.$(100.0), 211.1 (85.9), 196.1 (17.7), 165.1 (25.7), 115.1 (14.4)); Exact mass calc'd. for $\mathrm{C}_{16} \mathrm{H}_{18} \mathrm{O}_{2}: 242.1325$, found 242.1343.

8-Methoxy-4-(2-methoxymethyl-buta-1,3-dienyl)-1,2-dihydronaphthalene (36b): Compound 36a (165 mg, $0.68 \mathrm{mmol})$ was dissolved in dry THF $(20 \mathrm{~mL})$. $\mathrm{KO}^{\mathrm{t}} \mathrm{Bu}$ in THF $(3.4 \mathrm{~mL}, 1 \mathrm{M}, 3.4$ mmol) was added via syringe and stirred for 10 minutes. MeI $(378 \mu \mathrm{L}, 3.4 \mathrm{mmol})$ was added via syringe and the reaction stirred at room temperature for $3 \mathrm{~h}$. The reaction was quenched with water $(30 \mathrm{~mL})$ and extracted with diethyl ether $(2 \times 20 \mathrm{~mL})$. This afforded $\mathbf{3 6 b}$ as a yellow oil (168 mg, 99 \%). IR (neat); 2938 (s), 2836 (s), 1493 (s), 1248 (s), 1092 (s) cm ${ }^{-1}$; ${ }^{1} \mathrm{H}$ NMR $\left(\mathrm{CDCl}_{3}, 300 \mathrm{MHz}\right): \delta 7.12(\mathrm{~d}, J=4.0 \mathrm{~Hz}, 1 \mathrm{H}), 6.74-6.65(\mathrm{~m}, 3 \mathrm{H}), 6.37(\mathrm{~s}, 1 \mathrm{H}), 5.84(\mathrm{~d}, J=4.5$ $\mathrm{Hz}, 1 \mathrm{H}), 5.34$ (t, $J=18.8 \mathrm{~Hz}, 1 \mathrm{H}), 5.08(\mathrm{~d}, J=11.2 \mathrm{~Hz}, 1 \mathrm{H}), 4.22(\mathrm{~s}, 2 \mathrm{H}), 3.78$ (s, 3H), 3.40 (s, $3 \mathrm{H}), 2.76(\mathrm{t}, J=7.8 \mathrm{~Hz}, 2 \mathrm{H}), 2.35(\mathrm{~m}, 2 \mathrm{H}) ;{ }^{13} \mathrm{C} \mathrm{NMR}\left(\mathrm{CDCl}_{3}, 125 \mathrm{MHz}\right): \delta 158.8,137.9,135.9$, $133.2,130.0,127.9,127.4,125.5,114.4,113.8,111.0,74.0,57.9,55.2,28.5,23.2 ; \mathrm{MS}(\mathrm{EI}) \mathrm{m} / \mathrm{z}$ $256.1\left(\mathrm{M}^{+}\right.$(100.0), 225.1 (56.8), 211.1 (93.0), 171.1 (76.0), 115.1 (38.2), 45.0 (74.8)); Exact mass calc'd. for $\mathrm{C}_{17} \mathrm{H}_{20} \mathrm{O}_{2}$ : 256.1441, found 256.1413.

3,4-Dihydronaphthal-2-en-1-yl)buta-1,3-dien-2-methanol (39a): Compound 38 (415 mg, 2.26 $\mathrm{mmol})$ in cyclohexane $(5 \mathrm{~mL})$ and vinyl magnesium chloride $(4.0 \mathrm{~mL}, 1.7 \mathrm{M}, 6.78 \mathrm{mmol})$ were reacted together and the reaction quenched with $\mathrm{NH}_{4} \mathrm{Cl}(20 \mathrm{~mL})$. Chromatography (petroleum ether/ethyl acetate, 6:1) afforded 39a as a yellow oil (370 mg, 77\%). IR (neat); 3606 (s), 3448 (m), 3054 (s), 2987 (s), 2306 (m), 1422 (s) cm ${ }^{-1} ;{ }^{1} \mathrm{H}$ NMR $\left(\mathrm{CDCl}_{3}, 500 \mathrm{MHz}\right): \delta 7.17$ (s, 4H), 6.73 (ddd, $J=18.0 \mathrm{~Hz}, 11.3 \mathrm{~Hz}, 0.9 \mathrm{~Hz}, 1 \mathrm{H}), 6.49$ (s, 1H), 5.98 (td, $J=4.7 \mathrm{~Hz}, 1.6 \mathrm{~Hz}, 1 \mathrm{H}), 5.39$ $(\mathrm{dt}, J=18.0 \mathrm{~Hz}, 0.9 \mathrm{~Hz}, 1 \mathrm{H}), 5.13(\mathrm{dt}, J=11.3 \mathrm{~Hz}, 1.4 \mathrm{~Hz}, 1 \mathrm{H}), 4.48(\mathrm{~d}, J=1.1 \mathrm{~Hz}, 2 \mathrm{H}), 2.80(\mathrm{t}$, 
$J=7.7 \mathrm{~Hz}, 2 \mathrm{H}), 2.42-2.37(\mathrm{~m}, 2 \mathrm{H}), 2.20($ br s, $1 \mathrm{H}) ;{ }^{13} \mathrm{C} \mathrm{NMR}\left(\mathrm{CDCl}_{3}, 125 \mathrm{MHz}\right): \delta 138.7$, 135.9, 134.6, 133.4, 132.9, 129.8, 128.2, 127.4, 127.1, 126.4, 124.2, 114.2, 64.0, 27.8, 23.2; MS (EI) $\mathrm{m} / \mathrm{z} 212.1\left(\mathrm{M}^{+}\right.$(54.5), 181.1 (100.0), 165.1 (53.2), 141.1 (35.2), 128.1 (26.5)); Exact mass calc'd. for $\mathrm{C}_{15} \mathrm{H}_{16} \mathrm{O}: 212.1200$, found 212.1199 .

4-(2-Methoxymethyl-buta-1,3-dienyl)-1,2-dihydro-naphthalene (39b): Compound 39a (160 $\mathrm{mg}, 0.79 \mathrm{mmol})$ was dissolved in THF $(20 \mathrm{~mL})$. Sodium hydride $(60 \mathrm{mg}, 1.58 \mathrm{mmol})$ and methyl iodide $(0.24 \mathrm{~mL}, 3.95 \mathrm{mmol})$ were added and the solution stirred at room temperature for 14 hours. Water $(20 \mathrm{~mL})$ was added followed by diethyl ether $(3 \times 20 \mathrm{~mL})$ ectraction. Chromatography (petroleum ether/ethyl acetate, 20:1) afforded 39b as a colourless oil (155 mg, 87\%). IR ( $\left.\mathrm{CDCl}_{3}\right) ; 3161$ (m), 2936 (s), 2865 (s), 2814 (s), 1468 (m) cm ${ }^{-1} ;{ }^{1} \mathrm{H}$ NMR $\left(\mathrm{CDCl}_{3}, 300\right.$ MHz): $\delta 7.14$ (s, 4H), 6.70 (ddd, $J=17.9 \mathrm{~Hz}, 11.4 \mathrm{~Hz}, 0.9 \mathrm{~Hz}, 1 \mathrm{H}), 6.41$ (s, $1 \mathrm{H}), 5.98$ (td, $J=5.7$ $\mathrm{Hz}, 1.5 \mathrm{~Hz}, 1 \mathrm{H}), 5.39(\mathrm{~d}, J=17.8 \mathrm{~Hz}, 1 \mathrm{H}), 5.10(\mathrm{dt}, J=11.2 \mathrm{~Hz}, 1.5 \mathrm{~Hz}, 1 \mathrm{H}), 2.79(\mathrm{t}, J=6.7 \mathrm{~Hz}$, 2H), $2.42-2.35(\mathrm{~m}, 2 \mathrm{H}) ;{ }^{13} \mathrm{C}$ NMR $\left(\mathrm{CDCl}_{3}, 75 \mathrm{MHz}\right): \delta 136.4,135.1,133.9,133.4,130.3$, $130.2,127.9,127.5,126.8,124.7,115.0,74.4,58.4,28.3,23.6 ; \mathrm{MS}(\mathrm{EI}) \mathrm{m} / \mathrm{z} 226.1\left(\mathrm{M}^{+}(55.7)\right.$, 196.1 (38.0), 181.1 (100.0), 165.1 (74.7), 141.1 (39.2), 115.1 (41.0); Exact mass calc'd. for $\mathrm{C}_{16} \mathrm{H}_{18} \mathrm{O}: 226.1376$, found 226.1394 .

(Cyclohex-1-en-1-yl)-buta-1,3-dien-2-methanol (42a): Compound 41 (400 mg, $2.94 \mathrm{mmol}$ ) in cyclohexane $(5 \mathrm{~mL})$ and vinyl magnesium chloride $(5.53 \mathrm{~mL}, 1.7 \mathrm{M}, 8.64 \mathrm{mmol})$ were reacted together and the reaction quenched with saturated ammonium chloride $(20 \mathrm{~mL})$. Chromatography (petroleum ether/ethyl acetate, 6:1) afforded 42a as a yellow oil (433 mg, 90\%). IR (neat); 3338 (s), 2929 (s), 2858 (m), 1436 (m) cm ${ }^{-1} ;{ }^{1} \mathrm{H}$ NMR $\left(\mathrm{CDCl}_{3}, 500 \mathrm{MHz}\right)$ : $\delta 6.79$ (ddd, $J=17.9 \mathrm{~Hz}, 11.3 \mathrm{~Hz}, 0.8 \mathrm{~Hz}, 1 \mathrm{H}), 5.98$ (s, 1H), 5.65 (sextet, $J=2.0 \mathrm{~Hz}, 1 \mathrm{H}$ ), 5.29 (d, $J=17.9 \mathrm{~Hz}, 1 \mathrm{H}), 5.07$ (dt, $J=11.2 \mathrm{~Hz}, 1.4 \mathrm{~Hz}, 1 \mathrm{H}), 2.12-2.05(\mathrm{~m}, 4 \mathrm{H}), 1.90$ (br s, $1 \mathrm{H})$, $1.64-1.54(\mathrm{~m}, 4 \mathrm{H}) ;{ }^{13} \mathrm{C} \mathrm{NMR}\left(\mathrm{CDCl}_{3}, 125 \mathrm{MHz}\right): \delta 134.7,134.4,133.2,133.2,129.7,113.7$, 64.8, 29.1, 25.7, 22.7, 22.0; MS (EI) m/z $164.1\left(\mathrm{M}^{+}\right.$(17.0), 133.1 (32.9), 121.1 (25.2), 105.1 (28.1), 91.1 (100.0), 79.1 (37.8)); Exact mass calc'd. for $\mathrm{C}_{11} \mathrm{H}_{16} \mathrm{O}: 164.1189$, found 164.1177.

6-Methoxymethyl-1,2,3,4-tetrahydronaphthalene (43): Compound 42b (120 mg, $0.67 \mathrm{mmol})$ was dissolved in toluene $(20 \mathrm{~mL})$ and placed in a sealed tube. The solution was heated to $250{ }^{\circ} \mathrm{C}$ for 16 hours. The toluene was removed under reduced pressure. Chromatography (petroleum ether) afforded 43 as a colourless oil (28 mg, 23\%). IR (neat); 2936 (s), $2852(\mathrm{~m}), 2247$ (m), $1094(\mathrm{~m}), \mathrm{cm}^{-1} ;{ }^{1} \mathrm{H}$ NMR $\left(\mathrm{CDCl}_{3}, 300 \mathrm{MHz}\right): \delta 7.24(\mathrm{~s}, 3 \mathrm{H}), 4.36(\mathrm{~s}, 2 \mathrm{H}), 3.35(\mathrm{~s}, 3 \mathrm{H}), 2.74(\mathrm{~m}$, $4 \mathrm{H}), 1.09$ (s, 4H); ${ }^{13} \mathrm{C} \mathrm{NMR}\left(\mathrm{CDCl}_{3}, 75 \mathrm{MHz}\right): \delta 137.5,137.0,135.5,129.5,129.0,125.4,75.1$, 58.3, 29.7, 29.5, 23.6; MS (EI) m/z 176.1 (M+ 85.2), 145.1 (87.8), 129.1 (100.0), 115.1 (59.9), 91.1 (75.5)); Exact mass calc'd. for $\mathrm{C}_{12} \mathrm{H}_{16} \mathrm{O}: 176.1184$, found 176.1167 .

4-(2-Methoxymethyl-buta-1,3-dienyl)-1,2-dihydro-naphthalene (39b) : Alcohol 39a (160 mg, $0.79 \mathrm{mmol})$ was dissolved in THF $(20 \mathrm{~mL})$. Sodium hydride $(60 \mathrm{mg}, 1.58 \mathrm{mmol})$ and methyl iodide $(0.24 \mathrm{~mL}, 3.95 \mathrm{mmol})$ were added and the solution allowed to stir at room temperature for 14 hours. Water $(20 \mathrm{~mL})$ was added and aqueous extraction with diethyl ether $(3 \times 20 \mathrm{~mL})$ was performed. Purification by silica gel chromatography employing a 20:1 pet ether/ethyl acetate solvent system. This afforded $\mathbf{3 9 b}$ as a colourless oil $(155 \mathrm{mg}, 87 \%)$.

IR $\left(\mathrm{CDCl}_{3}\right) ; 3161(\mathrm{~m}), 2936$ (s), 2865 (s), 2814 (s), $1468(\mathrm{~m}) \mathrm{cm}^{-1} ;{ }^{1} \mathrm{H} \mathrm{NMR}\left(\mathrm{CDCl}_{3}, 300 \mathrm{MHz}\right)$ : $\delta 7.14(\mathrm{~s}, 4 \mathrm{H}), 6.70$ (ddd, $J=17.9 \mathrm{~Hz}, 11.4 \mathrm{~Hz}, 0.9 \mathrm{~Hz}, 1 \mathrm{H}), 6.41$ (s, 1H), 5.98 (td, $J=5.7 \mathrm{~Hz}$, 
$1.5 \mathrm{~Hz}, 1 \mathrm{H}), 5.39(\mathrm{~d}, J=17.8 \mathrm{~Hz}, 1 \mathrm{H}), 5.10(\mathrm{dt}, J=11.2 \mathrm{~Hz}, 1.5 \mathrm{~Hz}, 1 \mathrm{H}), 2.79$ (t, $J=6.7 \mathrm{~Hz}$, $2 \mathrm{H}), 2.42-2.35(\mathrm{~m}, 2 \mathrm{H}) ;{ }^{13} \mathrm{C}$ NMR $\left(\mathrm{CDCl}_{3}, 75 \mathrm{MHz}\right): \delta 136.4,135.1,133.9,133.4,130.3$, $130.2,127.9,127.5,126.8,124.7,115.0,74.4,58.4,28.3,23.6 ; \mathrm{MS}(\mathrm{EI}) \mathrm{m} / \mathrm{z} 226.1\left(\mathrm{M}^{+}\right.$(55.7), 196.1 (38.0), 181.1 (100.0), 165.1 (74.7), 141.1 (39.2), 115.1 (41.0); Exact mass calc'd. For $\mathrm{C}_{16} \mathrm{H}_{18} \mathrm{O}: 226.1376$. Observed: 226.1394.

9,10-Dihydrophenanthrene-3-carbaldehyde (44): 2,3-Dichloro-5,6-dicyano-1,4-benzoquinone (DDQ) $(26.0 \mathrm{mg}, 0.114 \mathrm{mmol}, 1.2$ equiv) was added to a solution of $\mathbf{3 9 b}(20.3 \mathrm{mg}, 0.095 \mathrm{mmol})$ in toluene $(0.637 \mathrm{~mL})$ and the mixture refluxed for $5 \mathrm{hrs}$. The mixture was diluted with water and the aqueous phase was extracted with diethyl ether $(3 \times 10 \mathrm{~mL})$. The combined organic layers were washed with water $(1 \times 10 \mathrm{~mL})$, brine $(1 \times 10 \mathrm{~mL})$ and dried over $\mathrm{MgSO}_{4}$. Chromatography (petroleum ether/ $\left.\mathrm{Et}_{2} \mathrm{O}, 9: 1\right)$ afforded $44(9.57 \mathrm{mg}, 48 \%)$ as a colourless liquid. IR (neat) 3058, $2930,2835,2728,1698,1607,1565,1491,1430,1296,1175,1094,1029,826,770 \mathrm{~cm}^{-1} ;{ }^{1} \mathrm{H}$ NMR $\left(\mathrm{CDCl}_{3}, 500 \mathrm{MHz}\right): \delta 10.03(\mathrm{~s}, 1 \mathrm{H}), 8.24(\mathrm{~d}, J=1.5 \mathrm{~Hz}, 1 \mathrm{H}), 7.83(\mathrm{~d}, J=7.7 \mathrm{~Hz}, 1 \mathrm{H})$, 7.72 (dd, $J=7.7,1.6,1 \mathrm{H}), 7.38$ (d, $J=7.7,1 \mathrm{H}) 7.35-7.32(\mathrm{~m}, 1 \mathrm{H}) 7.29-7.25$ (m, 2H) 2.96-2.87 (m, 4H). ${ }^{13} \mathrm{C} \mathrm{NMR}\left(\mathrm{CDCl}_{3}, 126 \mathrm{MHz}\right): \delta 192.1,144.6,137.2,135.7,135.5,133.3,128.9,128.9$, 128.3, 128.2, 127.3, 124.8, 124.0, 29.5, 28.4. Exact mass calc'd. for $\mathrm{C}_{16} \mathrm{H}_{16} \mathrm{O}: 208.0888$, found 208.0888 . 